\title{
Non-local representations of the ageing algebra in higher dimensions
}

\author{
Stoimen Stoimenov ${ }^{a}$ and Malte Henkel ${ }^{b}$ \\ ${ }^{a}$ Institute of Nuclear Research and Nuclear Energy, Bulgarian Academy of Sciences, \\ 72 Tsarigradsko chaussee, Blvd., BG - 1784 Sofia, Bulgaria \\ ${ }^{b}$ Groupe de Physique Statistique, Département de Physique de la Matière et des Matériaux, \\ Institut Jean Lamour (CNRS UMR 7198), Université de Lorraine Nancy, \\ B.P. 70239, F - 54506 Vandœuvre lès Nancy Cedex, France
}

\begin{abstract}
The ageing Lie algebra $\mathfrak{a g e}(d)$ and especially its local representations for a dynamical exponent $z=2$ has played an important rôle in the description of systems undergoing simple ageing, after a quench from a disordered state to the low-temperature phase. Here, the construction of representations of $\mathfrak{a g e}(d)$ for generic values of $z$ is described for any space dimension $d>1$, generalising upon earlier results for $d=1$. The mechanism for the closure of the Lie algebra is explained. The Lie algebra generators contain higher-order differential operators or the Riesz fractional derivative. Co-variant two-time response functions are derived. Some simple applications to exactly solvable models of phase separation or interface growth with conserved dynamics are discussed.
\end{abstract}




\section{Introduction}

Understanding the cooperative properties of strongly interacting many-body problems continues to pose many challenges. Here, we are interested in a particular class of non-equilibrium phenomena, usually referred to as 'ageing'. While first systematically studied in structural glasses quenched from a molten state to below the 'glass-transition temperature' 58, very similar phenomena have been found in many other glassy and non-glassy systems far from equilibrium, see e.g. [8, 23] for surveys and further references. Schematically, one may characterise ageing systems by (i) a slow relaxation dynamics, (ii) absence of time-translation-invariance and (iii) dynamical scaling. Therefore, if ageing is understood in this way, the study of the ageing of systems far from equilibrium gives physically well-motivated setting for the analysis of the consequences of dynamical scaling and the investigation of possible generalisations and extensions.

In this work, we shall be interested in the dynamical symmetries of ageing systems. We shall restrict throughout to what is known as 'simple ageing', where the dynamics can described in terms of a single time-dependent length $L(t)$ such that for large times $L(t) \sim t^{1 / z}$, which defines the dynamical exponent $z$. This growing length scale signals a natural dynamical scaling in the long-time limit $t \rightarrow \infty$. It has been proposed that it might be possible to enlarge this to a larger set of local scale transformation, to be referred to as 'local scale-invariance' (LSI). The current state of LSI-theory, with its explicit predictions for two-time responses and correlators, has been recently reviewed in detail in [23, where also the available numerical tests as well as the respective exactly solved models are discussed 1 Here, we shall be concerned with certain algebraic techniques with the distant goal to extend known representations of LSI algebras with dynamical exponents $z=2$ (or $z=1$ ) to more generic values. As a first step in carrying out this programme, we shall study here certain non-local symmetries of some linear equations, and shall leave the much more difficult case of genuinely non-linear equations for future work.

The analysis of the ageing of several simple magnetic systems, without disorder nor frustrations, without any macroscopic conservation law of the dynamics, and undergoing ageing when quenched to a temperature $T<T_{c}$ below the critical temperature $T_{c}>0$ is characterised by the dynamical exponent $z=2$ [6]. Furthermore, it has been suggested that the detailed scaling form of the two-time correlators and responses can be obtained by an extension of simple dynamical scaling with $z=2$ towards a larger Lie group [16, 17, 18] of which the so-called 'ageing algebra' $\mathfrak{a g e}(d)=\left\langle X_{0,1}, Y_{ \pm \frac{1}{2}}^{(i)}, M_{0}, R_{i j}\right\rangle_{1<i<j<d}$ is the Lie algebra. It can be defined in terms of its non-vanishing commutators [20, 21]

$$
\begin{aligned}
& {\left[X_{n}, Y_{m}^{(i)}\right]=\left(\frac{n}{2}-m\right) Y_{n+m}^{(i)}, \quad\left[X_{n}, X_{n^{\prime}}\right]=\left(n-n^{\prime}\right) X_{n+n^{\prime}}, \quad\left[Y_{\frac{1}{2}}^{(i)}, Y_{-\frac{1}{2}}^{(j)}\right]=\delta_{i j} M_{0},} \\
& {\left[R_{i j}, R_{k \ell}\right]=\delta_{i \ell} R_{j k}+\delta_{j k} R_{i \ell}-\delta_{i k} R_{j \ell}-\delta_{j \ell} R_{i k}, \quad\left[R_{i j}, Y_{m}^{(k)}\right]=\delta_{j k} Y_{m}^{(i)}-\delta_{i k} Y_{m}^{(j)}}
\end{aligned}
$$

with $n, n^{\prime}=0,1, m= \pm \frac{1}{2}$ and $1 \leq i \leq j \leq d$. When acting on space-time coordinates $(t, \boldsymbol{r})$, a

\footnotetext{
${ }^{1}$ One important consequence of LSI is that for non-equilibrium scaling, far from the stationary state, each scaling operator is characterised by two independent scaling exponents, labelled $x$ and $\xi$ in eqs. (1.2) and (1.7) below. While allowing for $\xi \neq 0$ is certainly required in certain exactly solved systems such as the $1 D$ GlauberIsing quenched to temperature $T=0$ and certainly improves the fit with the numerical data, it also appears that the agreement is not perfect. Very recently, it has been attempted to explain remaining subtle differences in the scaling of the two-time responses $R(t, s)$ in several non-equilibrium models with $z<2$ terms of a 'logarithmic' extension of LSI [26, 27, see 28] for a short review.
} 
representation of (1.1) in terms of affine differential operators is:

$$
\begin{aligned}
X_{0} & =-t \partial_{t}-\frac{1}{2}\left(\boldsymbol{r} \cdot \partial_{\boldsymbol{r}}\right)-\frac{x}{2} & & \text { dilatation } \\
X_{1} & =-t^{2} \partial_{t}-t\left(\boldsymbol{r} \cdot \partial_{\boldsymbol{r}}\right)-\frac{\mathcal{M}}{2} \boldsymbol{r}^{2}-(x+\xi) t & & \text { special transformation } \\
Y_{-\frac{1}{2}}^{(i)} & =-\partial_{r_{i}} & & \text { space-translations } \\
Y_{\frac{1}{2}}^{(i)} & =-t \partial_{r_{i}}-\mathcal{M} r_{i} & & \text { Galilei-transformation } \\
M_{0} & =-\mathcal{M} & & \text { phase shift } \\
R_{i j} & =r_{i} \partial_{r_{j}}-r_{j} \partial_{r_{i}}=-R_{j i}, & & \text { rotations. }
\end{aligned}
$$

together with the physical interpretation of these generators. Clearly, the representation (1.2) has a dynamical exponent $z=2$ and acts locally on the space-time coordinates. Furthermore, it generates a set of dynamical symmetries of the Schrödinger (or diffusion) equation:

$$
\mathcal{S} \phi(t, \boldsymbol{r})=\left(2 \mathcal{M} \partial_{t}+\frac{2 \mathcal{M}}{t}(x+\xi-d / 2)-\nabla_{\boldsymbol{r}}^{2}\right) \phi(t, \boldsymbol{r})=0,
$$

in the sense that each of the generators of $\mathfrak{a g} \mathfrak{e}(d)$ maps a solution of (1.3) onto another solution. Herein, $(\mathcal{M}, x, \xi)$ is a triplet of constants which together characterise the solution $\phi=\phi_{(\mathcal{M}, x, \xi)}$ of this equation. Physically, $\mathcal{M}$ is interpreted as an inverse diffusion constant, whenever $\mathcal{M} \in \mathbb{R}$ or else as a non-relativistic mass if $\mathcal{M}=\mathrm{i} m$ with $m \in \mathbb{R}$. Furthermore, $x$ and $\xi$ are the two independent scaling dimensions which characterise the field $\phi=\phi_{(\mathcal{M}, x, \xi)}$

For systems undergoing simple ageing with $z=2$, the extended local scale-invariance as described by the representation (1.2) of $\mathfrak{a} \mathfrak{g} \mathfrak{e}(d)$ indeed seems to give an appropriate description, including several exactly solved examples where $\xi \neq 0$ is required [20, 23] - the best-known example is the $1 D$ Glauber-Ising model quenched to $T=0$. The main prediction concerns the derivation of two-time (linear) responses $R=R(t, s)=\left.\frac{\delta\langle\phi(t)\rangle}{\delta h(s)}\right|_{h=0}$ of the order parameter $\phi$ with respect to its conjugate magnetic field, from the assumption of co-variance under the chosen representation of $\mathfrak{a g e}(d)$. The derivation is based on the Janssen-de Dominicis theory [31] which permits to re-express responses $R(t, s)=\langle\phi(t) \widetilde{\phi}(s)\rangle$ as a correlator of the order parameter $\phi=\phi_{(\mathcal{M}, x, \xi)}$ and the conjugate 'response field' $\widetilde{\phi}=\widetilde{\phi}_{(-\mathcal{M}, \widetilde{x}, \widetilde{\xi})}$ (see e.g. [31, 60, 61] for introductions and detailed references). Co-variance under $\mathfrak{a g e}(d)$ then leads to a set of linear partial differential equations for $R(t, s) 3$ In statistical physics, a common formulation uses a

\footnotetext{
${ }^{2}$ If one were to add the time-translations $X_{-1}=-\partial_{t}$, one would obtain the embedding $\mathfrak{a g} \mathfrak{g}(d) \subset \mathfrak{a g e}(d) \oplus$ $\mathbb{C} X_{-1} \hookrightarrow \mathfrak{s c h}(d)$ into the Schrödinger algebra $\mathfrak{s c h}(d)$, with (1.1) extended to $n=-1$. In the representation (1.2) one then has $\xi=0$. Invariance of (1.3) implies further that $x=d / 2$, a fact essentially known to Jacobi and Lie in 1843 and 1881, respectively and since then re-discovered many times.

Since time-translation-invariance is absent by definition in ageing systems and hence a stationary state is never reached, the new scaling dimension $\xi$ is a further universal characteristics of the relaxation process. The transformation $\phi(t, r)=t^{\xi_{\phi}} \Phi(t, \boldsymbol{r})$ maps an $\mathfrak{a g e}(d)$-quasi-primary scaling operator $\phi$ with the scaling dimensions $\left(x_{\phi}, \xi_{\phi}\right)$ to the $\mathfrak{s c h}(d)$-quasi-primary scaling operator $\Phi$ with scaling dimensions $\left(x_{\Phi}=x_{\phi}+2 \xi_{\phi}, \xi_{\Phi}=0\right)$ and confirmed for two-point functions [20]. The relationship between three-point and higher correlators is more involved 43 .

${ }^{3}$ There is a systematic way to 'dualise' the parameter $\mathcal{M}$ which permits to further extend age $(d)$-covariance to the covariance under a parabolic sub-algebra of the conformal algebra $\operatorname{conf}(d+2)$ in $d+2$ dimensions,
} 
stochastic Langevin equation

$$
\partial_{t} \phi(t, \boldsymbol{r})=-D \frac{\delta \mathcal{H}[\phi]}{\delta \phi(t, \boldsymbol{r})}+\eta(t, \boldsymbol{r})
$$

with a Ginzburg-Landau functional $\mathcal{H}$ and a centred gaussian noise $\eta$ with a $\delta$-correlated second moment. In the standard Janssen-de Dominicis formalism, this is related to the equation of motion derived from the dynamic functional

$$
\mathcal{J}[\widetilde{\phi}, \phi]=\int \mathrm{d} t \mathrm{~d} \boldsymbol{r}\left[\widetilde{\phi}\left(2 \mathcal{M} \partial_{t}-\Delta_{\boldsymbol{r}}\right) \phi+\widetilde{\phi} \frac{\delta \mathcal{V}[\phi]}{\delta \phi}-T \widetilde{\phi}^{2}\right]-\frac{1}{2} \int \mathrm{d} \boldsymbol{r} \mathrm{d} \boldsymbol{r}^{\prime} \widetilde{\phi}_{0}(\boldsymbol{r}) a\left(\boldsymbol{r}-\boldsymbol{r}^{\prime}\right) \widetilde{\phi}_{0}\left(\boldsymbol{r}^{\prime}\right)
$$

where $D^{-1}=2 \mathcal{M}, \mathcal{V}$ is the 'potential' part of the Ginzburg-Landau functional, $a(\boldsymbol{r})$ the correlator in the initial state $\widetilde{\phi}_{0}(\boldsymbol{r})=\widetilde{\phi}(0, \boldsymbol{r})$ and $T$ the temperature of the external heat bath. In the case of a quadratic potential $\mathcal{V}$, this action produces two equations of motion which both have a form similar to (1.2), and with the above characterisations of $\phi$ and $\widetilde{\phi}$ [47]. In this case, one has a decomposition $\mathcal{J}[\widetilde{\phi}, \phi]=\mathcal{J}_{0}[\widetilde{\phi}, \phi]+\mathcal{J}_{b}[\widetilde{\phi}]$ such that the 'deterministic part' $\mathcal{J}_{0}$ is invariant under the action of the Galilei sub-algebra $\mathfrak{g a l}(d)=\left\langle Y_{ \pm \frac{1}{2}}^{(i)}, M_{0}, R_{i j}\right\rangle_{1 \leq i<j \leq d}$. This implies the Bargman super-selection rules [2]. From this, it follows that [47, 23]

1. the computation of all response functions can be exactly reduced to the computation of response in the 'noiseless' theory governed by the functional $\mathcal{J}_{0}$, whose averages are given by co-variance conditions under the dynamical symmetry algebra

2. all correlators can be exactly reduced to certain integrals over higher response functions of the 'noiseless' theory

These calculations have been carried out for a long list of models undergoing simple ageing with $z=2$ [3, 9, 11, 23]. In spite of many encouraging numerical results, there is not yet a satisfactory analytical treatment available for a non-quadratic potential $\mathcal{V}$, where the main difficulty come from the non-trivial co-factors of the scaling operators $\phi, \widetilde{\phi}$ under Galilei- and special transformations $\left(Y_{\frac{1}{2}}, X_{1}\right)$. A way around this problem might be to perform first a dualisation with respect to $\mathcal{M}$, which converts the projective representation (1.2) of the nonsemi-simple Lie algebra $\mathfrak{a g e}(d)$ into a true representation of a sub-algebra of $\mathfrak{c o n \mathfrak { f }}(d+2)$ [56, 57].

Can one extend this procedure, at least for linear stochastic Langevin equations of motion, to arbitrary values of the dynamical exponent $z$ ? If we were to restrict to locally realised algebras, the recent classification of the non-relativistic limits of the conformal algebra [10] would only admit the cases (i) $z=1$ : the conformal algebra conf $(d)$ or the conformal Galilean algebra $\mathrm{CGA}(d)$ [15, 17, 45], eventually with the exotic central extension for $d=2$ [36] (ii) $z=2$ : the Schrödinger algebra and (iii) $z=\infty$; along with their sub-algebras. Further examples can only be found when looking at non-local representation, which means that we must go beyond the setting for first-order linear (affine) differential operators in the generators and must look for different, non-local realisations of the known abstract algebras. Some partial information is already available to serve as a guide:

which gives an algebraic way to derive the causality condition $t>s$ necessary for the interpretation of the covariant two-point function as a physical response [19, 25]. In this dualised form, the non-relativistic AdS/CFT correspondence becomes evident, see e.g. [1, 42, 54, 14. 
1. the Galilei-invariance of the non-relativistic equation of motion $\mathcal{S} \phi=0$ should be kept, which means that algebraically one should require

$$
\left[Y_{\frac{1}{2}}^{(i)}, Y_{-\frac{1}{2}}^{(j)}\right]=\delta_{i j} M_{0}, \quad\left[\mathcal{S}, Y_{ \pm \frac{1}{2}}^{(j)}\right]=\lambda_{ \pm}^{(j)} \mathcal{S}
$$

where the scalars $\lambda_{ \pm}^{(j)}$ are to be determined. For example, in sub-representations where $M_{0}=0$, this can be extended to a Schrödinger algebra representation for any value of $z$ [45, which describes massless particles, but with a rather trivial equation of motion, see below.

2. In the context of LSI, different realisations of generalised symmetry algebras have been constructed by using certain fractional derivatives [18, 22, 23]. The closure of these sets of generators can only be achieved by taking a quotient with respect to a certain set of 'physical' states. Although this has been successfully applied to certain physical models [4, 9] the closing procedure is not completely determined and it is not clear how to obtain the group (finite) transformations.

3. There are many physically well-motivated systems, such as the Kardar-Parisi-Zhang equation in $1 D$ with a dynamical exponent $z=3 / 2$ [33], diffusion-limited erosion [35] or else certain interacting particle-reaction models [55, 49] with $z=1$ and a non-local equation of motion. One would like to be able to discuss their dynamical symmetries in a systematic way, but these cases do not seem to be included in the list of known representations of known algebras.

A distinct and potentially more promising method has been explored in [24]. Therein, new non-local representations of $\mathfrak{a g e}(1)$ for an integer-valued dynamical exponent $z=n \in \mathbb{N}$ were constructed. They read

$$
\begin{aligned}
X_{0} & =-\frac{n}{2} t \partial_{t}-\frac{1}{2} r \partial_{r}-\frac{x}{2} \\
X_{1} & =\left(-\frac{n}{2} t^{2} \partial_{t}-t r \partial_{r}-(x+\xi) t\right) \partial_{r}^{n-2}-\frac{1}{2} \mu r^{2} \\
Y_{-\frac{1}{2}} & =-\partial_{r} \\
Y_{\frac{1}{2}} & =-t \partial_{r}^{n-1}-\mu r \\
M_{0} & =-\mu .
\end{aligned}
$$

The commutation relations (1.1) are indeed satisfied, but with a notable exception, namely

$$
\left[X_{1}, Y_{\frac{1}{2}}\right]=\frac{n-2}{2} t^{2} \partial_{r}^{n-3} \mathcal{S}
$$

where the 'Schrödinger equation' takes the form

$$
\mathcal{S} \psi(t, r):=\left(z \mu \partial_{t}-\partial_{r}^{z}+\frac{2 \mu}{t}\left(x+\xi-\frac{z-1}{2}\right)\right) \psi(t, r)=0,
$$

Therefore, the algebra is now closed only in the quotient space over solutions of eq. (1.9) and in addition the representations (1.7) act as dynamical symmetries of the same equation [24]. 
In this paper, we shall show how to generalise this construction (1.7) to any spatial dimension $d \geq 1$ and to dynamical exponents $z \geq 2$; which will be important in order to apply it to many physically relevant models. In contrast to the local case $z=2$, the passage to $d>1$ is not trivial. We shall describe this in section 2. Co-variant two-point functions are computed from these nonlocal representations in section 3, first for the case when $z$ is even (with special attention given to the case $z=4$ ) and afterwards for generic values of $z$, when the Riesz fractional derivative will play an important rôle. In section 4, we shall apply these results to some simple physical models, namely the kinetic spherical model with a conserved order-parameter and quenched to $T=T_{c}$ and the Mullins-Herring (or Wolf-Villain) equations of interface growth with mass conservation. The full space-time response are calculated from the non-local representations of $\mathfrak{a g e}(d)$ and the results will be compared with the known exact results [34, 38, 53, 4]. We conclude in section 5 .

\section{$2 \quad$ Non-local representations of $\mathfrak{a g e}(d)$}

As we shall now show, the non-local representations of $\mathfrak{a g e}(d)$ are quite different for even and generic values of the dynamical exponent $z$. We shall discuss these cases separately.

\subsection{Representations of $\mathfrak{a g e}(d)$ with an even dynamical exponent}

Consider the following representations of $\mathfrak{a g e}(d)$ for an even dynamical exponent $z=2 n$ :

$$
\begin{aligned}
Y_{-1 / 2}^{(i)} & :=-\partial_{i} \\
Y_{+1 / 2}^{(i)} & :=-t \partial_{i} \Delta^{n-1}-\mu r_{i} \\
X_{0} & :=-n t \partial_{t}-\frac{1}{2}\left(\boldsymbol{r} \cdot \partial_{\boldsymbol{r}}\right)-\frac{x}{2} \\
X_{1} & :=\left(-n t^{2} \partial_{t}-t\left(\boldsymbol{r} \cdot \partial_{\boldsymbol{r}}\right)-(x+\xi) t\right) \Delta^{n-1}-\frac{\mu}{2} \boldsymbol{r}^{2} \\
M_{0} & :=-\mu
\end{aligned}
$$

where $\partial_{i}=\partial / \partial r_{i}, i=1,2, \ldots, d$ and $\Delta=\Delta_{r}=\partial_{i} \partial_{i}$ is the Laplacian. Upon setting $n=1$, one recovers (1.2). The spatial rotations $R_{i j}$ are unchanged with respect to (1.2). In order to check the commutation relations (1.1) we use the following relations:

$$
\begin{aligned}
& {\left[\Delta^{n}, r_{i}\right]=2 n \partial_{i} \Delta^{n-1}} \\
& {\left[\Delta^{n}, \boldsymbol{r}^{2}\right]=4 n \boldsymbol{r} \cdot \partial_{\boldsymbol{r}} \Delta^{n-1}+2 n(d+2(n-1)) \Delta^{n-1} .}
\end{aligned}
$$

Then the commutators (1.1) are indeed satisfied, except the following:

$$
\left[X_{1}, Y_{\frac{1}{2}}^{(i)}\right]=(n-1) t^{2} \partial_{i} \Delta^{n-2} \mathcal{S}
$$

where

$$
\mathcal{S}=2 n \mu \partial_{t}-\Delta^{n}+\frac{2 \mu}{t}\left(x+\xi-\frac{d+2 n-2}{2}\right),
$$


that is the generators (2.1) closed into the Lie algebra $\mathfrak{a g e}(d)$ only over functions from the solution space of the "Schrödinger" equation

$$
\mathcal{S} \Phi(t, \boldsymbol{r})=\left(2 n \mu \partial_{t}-\Delta^{n}+\frac{2 \mu}{t}\left(x+\xi-\frac{d+2 n-2}{2}\right)\right) \Phi(t, \boldsymbol{r})=0 .
$$

The representation (2.1) acts as a dynamical symmetry of the same equation. Since (2.5) is linear, it is enough to check the following commutators

$$
\begin{aligned}
& {\left[\mathcal{S}, Y_{-\frac{1}{2}}^{(i)}\right]=\left[\mathcal{S}, Y_{\frac{1}{2}}^{(i)}\right]=\left[\mathcal{S}, M_{0}\right]=\left[\mathcal{S}, R_{i j}\right]=0} \\
& {\left[\mathcal{S}, X_{0}\right]=-n \mathcal{S}, \quad\left[\mathcal{S}, X_{1}\right]=-2 \operatorname{tn} \Delta^{n-1} \mathcal{S}}
\end{aligned}
$$

Hence for any solution of $\mathcal{S} \Phi=0$, the infinitesimal change $\delta \Phi=\mathcal{X} \mathcal{S} \Phi$ with a generator $\mathcal{X}$ chosen from (2.1) also solves the same equation.

\subsection{Representations of $\mathfrak{a g e}(d)$ with a generic dynamical exponent}

For generic values of the dynamical exponent $z$, an explicit representation of $\mathfrak{a g} \mathfrak{g}(d)$ is more difficult to find and turns out to be quite distinct from those found for an even-valued dynamical exponent. In particular, when the dynamical exponent is an odd number, a generalisation of the one-dimensional case in manner similar to z-even case is possible only for a sub-algebra $\left\{X_{0}, X_{1}, Y_{ \pm \frac{1}{2}}^{i}, M_{0}\right\}$ which does not include the rotations. In addition this algebra is closed over solution of the system of $d$ equations, which are not invariant under entire algebra. We shall not develop this case here.

Instead, in order to treat the case with a generic value of $z$, recall first the definition of the Riesz fractional derivative, see e.g. [41, 52, 48, 37, 23] for further details. This is a linear operator $\nabla_{r}^{\alpha}$ acting as follows

$$
\nabla_{\boldsymbol{r}}^{\alpha} f(\boldsymbol{r})=\mathrm{i}^{\alpha} \int_{\mathbb{R}^{d}} \frac{\mathrm{d} \boldsymbol{k}}{(2 \pi)^{d}}|\boldsymbol{k}|^{\alpha} e^{\mathrm{i} \boldsymbol{r} \cdot \boldsymbol{k}} \widehat{f}(k),
$$

where the right-hand side as to be understood in a distribution sense and $\widehat{f}(k)$ denotes the Fourier transform. Some elementary properties are, see e.g. [23]

$$
\begin{aligned}
& \nabla_{\boldsymbol{r}}^{\alpha} \nabla_{\boldsymbol{r}}^{\beta}=\nabla_{\boldsymbol{r}}^{\alpha+\beta} \\
& \nabla_{\boldsymbol{r}}^{2}=\sum_{i=1}^{d} \partial_{i}^{2}=\Delta_{r} \\
& {\left[\nabla_{\boldsymbol{r}}^{\alpha}, r_{i}\right]=\alpha \partial_{i} \nabla_{\boldsymbol{r}}^{\alpha-2}} \\
& {\left[\nabla_{\boldsymbol{r}}^{\alpha}, \boldsymbol{r}^{2}\right]=2 \alpha\left(\boldsymbol{r} \cdot \partial_{\boldsymbol{r}}\right) \nabla_{\boldsymbol{r}}^{\alpha-2}+\alpha(d+\alpha-2) \nabla_{\boldsymbol{r}}^{\alpha-2}} \\
& \nabla_{\mu \boldsymbol{r}}^{\alpha} f(\mu \boldsymbol{r})=|\mu|^{-\alpha} \nabla_{\boldsymbol{r}}^{\alpha} f(\mu \boldsymbol{r})
\end{aligned}
$$

We emphasise that the Riesz fractional derivative acts in many ways as a 'square root' of the Laplacian, see eq. (2.9).

Now consider the generators 


$$
\begin{aligned}
Y_{-1 / 2}^{(i)} & :=-\partial_{i} \\
Y_{+1 / 2}^{(i)} & :=-t \partial_{i} \nabla_{\boldsymbol{r}}^{z-2}-\mu r_{i} \\
X_{0} & :=-\frac{z}{2} t \partial_{t}-\frac{1}{2}\left(\boldsymbol{r} \cdot \partial_{\boldsymbol{r}}\right)-\frac{x}{2} \\
X_{1} & :=\left(-\frac{z}{2} t^{2} \partial_{t}-t\left(\boldsymbol{r} \cdot \partial_{\boldsymbol{r}}\right)-(x+\xi) t\right) \nabla_{\boldsymbol{r}}^{z-2}-\frac{\mu}{2} \boldsymbol{r}^{2} \\
M_{0} & :=-\mu \\
R_{i j} & :=r_{i} \partial_{j}-r_{j} \partial_{i}=-R_{j i}
\end{aligned}
$$

Using the properties (2.10 2.11) the commutators (1.1) are seen to hold true, except for

$$
\left[X_{1}, Y_{\frac{1}{2}}^{(i)}\right]=\frac{z-2}{2} t^{2} \partial_{i} \nabla_{r}^{z-4} \mathcal{S}
$$

where

$$
\mathcal{S}=z \mu \partial_{t}-\nabla_{r}^{z}+\frac{2 \mu}{t}\left(x+\xi-\frac{d+z-2}{2}\right)
$$

Hence, the generators (2.13) close into a Lie algebra on the quotient with respect to solutions of the equation

$$
\mathcal{S} \Phi(t, \boldsymbol{r})=\left(z \mu \partial_{t}-\nabla_{\boldsymbol{r}}^{z}+\frac{2 \mu}{t}\left(x+\xi-\frac{d+z-2}{2}\right)\right) \Phi(t, \boldsymbol{r})=0 .
$$

As before, the representation (2.13) generates dynamical symmetries of the equation (2.16) which can be seen from the commutators

$$
\begin{aligned}
& {\left[\mathcal{S}, Y_{-\frac{1}{2}}^{(i)}\right]=\left[\mathcal{S}, Y_{\frac{1}{2}}^{(i)}\right]=\left[\mathcal{S}, M_{0}\right]=\left[\mathcal{S}, R_{i j}\right]=0} \\
& {\left[\mathcal{S}, X_{0}\right]=-\frac{z}{2} \mathcal{S}, \quad\left[\mathcal{S}, X_{1}\right]=-z t \nabla_{r}^{z-2} \mathcal{S} .}
\end{aligned}
$$

Some comments are in order:

1. because of (2.9), the generators (2.1) for an even dynamical exponent $z=2 n$ are included as well, with the same invariant differential equation (2.16).

2. only if one adopts the correspondence $\partial_{r} \mapsto \nabla_{\boldsymbol{r}}$ between the partial derivative with respect to the $1 D$ spatial coordinate $r$ and the Riesz fractional derivative $\nabla_{\boldsymbol{r}}$, one has a relationship between the $1 D$ representation (1.7) and the representation (2.13) for $d>1$ dimensions.

3. the non-locality only enters into the Galilei- and special transformations, generated by $Y_{\frac{1}{2}}^{(i)}$ and $X_{1}$.

4. certainly, the choice of the Riesz fractional derivative is merely motivated by its convenient algebraic properties. It is still unknown whether this is an appropriate choice for the treatment of physical systems. 
Summarising, the representation of $\mathfrak{a g} \mathfrak{e}(d)$ proposed here explicitly uses generators acting nonlocally on space. In Fourier space, the form proposed here will lead to local, but non-analytic generators 4 The special case of an even-valued dynamical exponent appears to have rather special and possibly non-generic properties.

In addition, we have shown here that certain equations of motion can be said to posses the kind of non-local symmetry considered here, at the price of somewhat weakening the meaning of the term "dynamical symmetry" with respect to how it had been used for the cases where $z=1$ or $z=2$ admit generators acting locally. It remains an open problem how to reformulate this kind of non-local symmetry in terms of an invariance of a field-theoretic action.

\section{Co-variant two-point functions}

The two-point function

$$
F\left(t_{1}, t_{2}, \boldsymbol{r}_{1}, \boldsymbol{r}_{2}\right)=\left\langle\Phi_{1}\left(t_{1}, \boldsymbol{r}_{1}\right) \Phi_{2}\left(t_{2}, \boldsymbol{r}_{2}\right)\right\rangle
$$

can be derived from the covariance under representation (2.13) discussed in the previous section. Here

$$
\boldsymbol{r}_{1}=\left(r_{11}, \ldots, r_{1 i}, \ldots r_{1 d}\right), \quad \boldsymbol{r}_{2}=\left(r_{21}, \ldots, r_{2 i}, \ldots r_{2 d}\right) .
$$

Later, we shall mainly concentrate on the case $z=4$, relevant for the physical applications we have in mind (see section 4), but for the moment $z$ will be left arbitrary. As usual in conformal or local scale-invariance, one forms from (2.13) two-particle operators and co-variance then gives the system $\mathcal{X}_{i}(2) F=0$.

1. The co-variance under space-translations requires:

$$
Y_{-\frac{1}{2}}^{(i)}:\left(\partial_{r_{1 i}}+\partial_{r_{2 i}}\right) F=\left(\partial_{r_{i}}-\partial_{r_{i}}\right) F=0, \quad r_{i}=r_{1 i}-r_{2 i}
$$

Letting $\boldsymbol{r}=\left(r_{1}, \ldots, r_{i}, \ldots r_{d}\right)$, it follows that $F=F\left(t_{1}, t_{2}, \boldsymbol{r}\right)$.

2. The covariance under a "mass" transformations gives the Bargman super-selection rule $\mu_{1}+\mu_{2}=0$ and consequently only fields with opposite "masses" will give a non-trivial result for the two-points function. It follows that

$$
\Phi_{1}:\left(\mu ; x_{1}, \xi_{1}\right), \quad \Phi_{2}:\left(-\mu ; x_{2}, \xi_{2}\right),
$$

where $x_{1}, x_{2}$ and $\xi_{1}, \xi_{2}$ are the scaling dimensions which characterise the corresponding nonstationary quasi-primary scaling operators. Consequently, the scaling operator $\Phi_{1}$ with a positive mass $\mu>0$ will be associated with the order parameter $\phi$ whereas the scaling operator $\Phi_{2}$ with a negative mass $-\mu<0$ is interpreted as the corresponding response operator $\widetilde{\phi}$ in the context of Janssen-de Dominic theory, see section 1.

3. Next we rewrite the two-point function as

$$
F=F(\tau, v, \boldsymbol{r}), \quad \tau:=t_{1}-t_{2}, v:=t_{1} / t_{2},
$$

\footnotetext{
${ }^{4}$ Of course, in the limit $z \rightarrow 2$ one simply returns to the standard local representation (1.2) of $\mathfrak{a g} \mathfrak{e}(d)$.
} 
and obtain from the three co-variance conditions $Y_{\frac{1}{2}}^{(i)} F=0$ for $i=1, \ldots, d, X_{0} F=0$ and $X_{1} F=0$ the equations (with an implicit sum over repeated indices $j=1, \ldots, d$ )

$$
\begin{aligned}
{\left[-\tau \partial_{r_{i}} \nabla_{\boldsymbol{r}}^{z-2}-\mu_{1} r_{i}\right] F } & =0 \\
{\left[-\frac{z}{2} \tau \partial_{\tau}-\frac{1}{2} r_{j} \partial_{r_{j}}-\frac{x_{1}+x_{2}}{2}\right] F } & =0 \\
{\left[\left(-\frac{z}{2} \tau^{2} \frac{v+1}{v-1} \partial_{\tau}-\frac{z}{2} \tau v \partial_{v}-\frac{\tau v}{v-1} r_{j} \partial_{r_{j}}-\left(x_{1}+\xi_{1}\right) \frac{\tau v}{v-1}-\left(x_{2}+\xi_{2}\right) \frac{\tau}{v-1}\right) \nabla_{r}^{z-2}\right.} & \\
\left.-\frac{\mu_{1}}{2} r_{i}^{2}\right] F & =0
\end{aligned}
$$

Acting with $\nabla_{\boldsymbol{r}}^{z-2}$ on (3.7), the difficult eq. (3.8) can be simplified to

$$
\nabla_{r}^{z-2}\left(\frac{z}{2} v \partial_{v}+\frac{v}{v-1}\left(x_{1}-x_{2}+2 \xi_{1}-2 n+2\right)+\frac{1}{v-1}\left(x_{2}-x_{1}+2 \xi_{2}-z+2\right)\right) F=0
$$

In analogy with the one-dimensional case [24, it is clear that each of the equations (3.6, 3.7 3.9. will fix the dependence of $F=F(\tau, v, \boldsymbol{r})$ on one of its variables. In fact, since the variable $v$ does not enter explicitly into eqs. (3.6.3.7), these two alone will completely fix the dependence of $F(\tau, v, \boldsymbol{r})$ on $\tau$ and $\boldsymbol{r}$ such that the dependence on $v$ factorises. Then the $(z-2)$-fold (fractional) derivatives in (3.9) can be dropped. We also checked that the closure condition of our representation is automatically satisfied, as it should be, and as seen for $d=1$ before [24]. Isolating the usual power-law pre-factor which only depends on the single time $t_{2}$, we find the following reduced form

$$
F=t_{2}^{-\left(x_{1}+x_{2}\right) / z}(v-1)^{-\frac{2}{z}\left[\left(x_{1}+x_{2}\right) / 2+\xi_{1}+\xi_{2}-z+2\right]} v^{-\frac{1}{z}\left[x_{2}-x_{1}+2 \xi_{2}-z+2\right]} f(\tau, \boldsymbol{r}) .
$$

where the last function $f(\tau, \boldsymbol{r})$ has to be determined from eqs. (3.6. 3.7).

4. The co-variance condition under each rotation $R_{i j}$ gives, for scalar quasi-primary operators $\Phi_{1,2}$

$$
\left(r_{i} \partial_{r_{j}}-r_{j} \partial_{r_{i}}\right) F=0
$$

and leads to $f=f(\tau, u)$, with $u=\boldsymbol{r}^{2}$ as expected.

Combining this with dynamical scaling eq. (3.7), one has simply

$$
f=f\left(u^{z / 2} \tau^{-1}\right)
$$

and the form of the last remaining function $f$ has to be found from Galilei-covariance, as given by (3.7).

This is the technically most demanding part of the calculation. For the sake of computational simplicity, we shall distinguish the cases of an even dynamical exponent $z=2 n$ (in particular, we shall describe below an explicit application of the case $z=4$ ) before we consider the case of generic values of $z$.

\subsection{Even dynamical exponent $z=2 n$}

Using the notation $p:=u^{z / 2} \tau^{-1}$, Galilei-covariance gives, for each $i=1, \ldots, d$

$$
\left(\tau \partial_{r_{i}} \Delta_{r}^{n-1}+\mu r_{i}\right) f(\tau, \boldsymbol{r})=r_{i}\left(2 \tau \partial_{u} \Delta_{u}^{n-1}+\mu\right) f(\tau, u)=r_{i}\left((2 n)^{n} p^{\frac{n-1}{n}} \partial_{p} \Delta_{p}^{n-1}+\mu\right) f(p)=0 .
$$


Here we use the following notations for the various forms of the Laplacian

$$
\begin{aligned}
\Delta_{r} & =\sum_{i=1}^{d} \frac{\partial^{2}}{\partial r_{i}^{2}}=: \Delta_{u}=2 d \partial_{u}+4 u \partial_{u}^{2} \\
& =\frac{2 n}{\tau^{1 / n}}\left((d+2(n-1)) p^{\frac{n-1}{n}} \partial_{p}+2 n p^{\frac{2 n-1}{n}} \partial_{p}^{2}\right)=: \frac{2 n}{\tau^{1 / n}} \Delta_{p}
\end{aligned}
$$

In contrast to $\Delta_{r}^{n-1}$ which is a formal power series of $\Delta_{r}$, the explicit forms of $\Delta_{u}^{n-1}$ and $\Delta_{p}^{n-1}$ must be calculated for any specific value of $n$. For example

$$
\Delta_{u}^{2} g(u)=\Delta_{u}\left(\Delta_{u} g(u)\right)=\left(4 d(d+2) \partial_{u}^{2}+16(d+2) u \partial_{u}^{3}+16 u^{2} \partial_{u}^{4}\right) g(u) .
$$

For illustration, we solve the equation (3.13) for $n=2$, that is $z=4$. In this case, one has

$$
\left((d+2) \partial_{p}+2(d+8) p \partial_{p}^{2}+8 p^{2} \partial_{p}^{3}+\frac{\mu}{8}\right) f(p)=0
$$

There are three independent solutions which are sought as Frobénius series

$$
f(p)=\sum_{k=0}^{\infty} a_{k} p^{k+\sigma}, a_{0} \neq 0 .
$$

where the exponent $\sigma$ is found from the characteristic equation $\sigma\left(8 \sigma^{2}+2 \sigma(d-4)+2-d\right)=0$

with solutions $\sigma=0, \frac{1}{2}, \frac{1}{2}-\frac{d}{4}$. Standard methods give a recursion relation for the coefficients $a_{m}$ and lead to, for $n=2$ or equivalently $z=4$

$$
\begin{aligned}
f(p)= & f_{0}{ }_{0} F_{2}\left(\frac{1}{2}, \frac{1}{2}+\frac{d}{4} ;-\frac{\mu p}{64}\right)+f_{1} p^{1 / 2}{ }_{0} F_{2}\left(\frac{3}{2}, \frac{d}{4}+1 ;-\frac{\mu p}{64}\right) \\
& +f_{2} p^{1 / 2-d / 4}{ }_{0} F_{2}\left(1-\frac{d}{4}, \frac{3}{2}-\frac{d}{4} ;-\frac{\mu p}{64}\right) .
\end{aligned}
$$

where ${ }_{0} F_{2}$ is a generalised hyper-geometric function and $f_{0,1,2}$ are normalisation constants. One could use the explicitly known asymptotic forms of ${ }_{0} F_{2}(a, b ; x)$ for $x \rightarrow \pm \infty$ in order to identify the sub-space of solutions which do not diverge for $|\mu p| \gg 1$ [63, 24], but we shall not need this explicitly later.

\subsection{Generic dynamical exponent}

The covariance under the generalised Galilei transformations of the representation (2.13) requires the $d$ conditions

$$
\left(\tau \partial_{r_{j}} \nabla_{r}^{z-2}+\mu r_{j}\right) f(\tau, \boldsymbol{r})=0, j=1, \ldots, d .
$$

The Frobénius series method from above cannot be used, because of the fractional derivative. However, eq. (3.19) can be solved in Fourier space where

$$
f(\tau, \boldsymbol{r})=\frac{1}{(2 \pi)^{d}} \int_{\mathbb{R}^{d}} \mathrm{~d} \boldsymbol{k} e^{\mathrm{i} \boldsymbol{k} \cdot \boldsymbol{r}} \widehat{f}(\tau, \boldsymbol{k})
$$


This leads to the correspondences $\partial_{j} \mapsto \mathrm{i} k_{j}, r_{j} \mapsto \mathrm{i} \frac{\partial}{\partial k_{j}}$ and $\nabla_{\boldsymbol{r}}^{\alpha} \mapsto(\mathrm{i}|\boldsymbol{k}|)^{\alpha}$. It follows that equation (3.19) takes the following form in Fourier space:

$$
\left(\mu \partial_{k_{j}}+\mathrm{i}^{z-2} \tau k_{j}|\boldsymbol{k}|^{z-2}\right) \widehat{f}(\tau, \boldsymbol{k})=0, \quad j=1, \ldots, d
$$

and has the the solution

$$
\widehat{f}(\tau, \boldsymbol{k})=f_{0}(\tau) \exp \left[-\frac{\mathrm{i}^{z-2}}{z} \frac{\tau}{\mu}|\boldsymbol{k}|^{z}\right] .
$$

This is rewritten in the direct space as follows

$$
f(\tau, \boldsymbol{r})=\frac{f_{0}(\tau)}{(2 \pi)^{d}} \int_{\mathbb{R}^{d}} \mathrm{~d} \boldsymbol{k} \exp \left[\mathrm{i} \boldsymbol{k} \cdot \boldsymbol{r}-\frac{\mathrm{i}^{z-2}}{z} \frac{\tau}{\mu}|\boldsymbol{k}|^{z}\right]=\frac{f_{0}(\tau)}{(2 \pi)^{d}} I_{\beta}(\boldsymbol{r}) ; \beta:=\frac{\mathrm{i}^{z-2} \tau}{z \mu}
$$

with the following abbreviation

$$
I_{\beta}(\boldsymbol{r}):=\int_{\mathbb{R}^{d}} \mathrm{~d} \boldsymbol{k} \exp \left[\mathrm{i} \boldsymbol{k} \cdot \boldsymbol{r}-\beta|\boldsymbol{k}|^{z}\right]
$$

In what follows, we shall admit $\beta \in \mathbb{C}$, but suitably restricted such that integral converges (i.e. $\operatorname{Re} \beta>0$ would be sufficient). To evaluate the integral (3.24) formally, introduce $d$-dimensional in spherical coordinates

$$
\begin{aligned}
k_{1} & =k \cos \theta_{1} \\
k_{2} & =k \sin \theta_{1} \cos \theta_{2} \\
\vdots & \\
k_{d-1} & =k \sin \theta_{1} \sin \theta_{2} \ldots \sin \left(\theta_{d-2}\right) \cos \left(\theta_{d-1}\right) \\
k_{d} & =k \sin \theta_{1} \sin \theta_{2} \ldots \sin \left(\theta_{d-2}\right) \sin \left(\theta_{d-1}\right),
\end{aligned}
$$

where $k=|\boldsymbol{k}|, \theta_{j} \in[0, \pi)$ for $j=1, \ldots d-2$ and $\theta_{d-1} \in[0,2 \pi)$. The jacobian is

$$
\frac{\partial\left(k_{1}, k_{2}, \ldots, k_{d}\right)}{\partial\left(k, \theta_{1}, \ldots, \theta_{d-1}\right)}=k^{d-1} \sin ^{d-2} \theta_{1} \sin ^{d-3} \theta_{2} \ldots \sin \left(\theta_{d-2}\right)
$$

Then the integral (3.24) becomes

$$
\begin{aligned}
I_{\beta}(\boldsymbol{r})= & \int_{0}^{\infty} \mathrm{d} k k^{d-1} e^{-\beta k^{z}} \int_{0}^{\pi} \mathrm{d} \theta_{1} e^{\mathrm{i} k r \cos \theta_{1}} \sin ^{d-2} \theta_{1} \\
& \times \int_{0}^{\pi} \mathrm{d} \theta_{2} \sin ^{(d-1)-2} \theta_{2} \ldots \int_{0}^{\pi} \mathrm{d} \theta_{d-2} \sin ^{(d-1)-(d-2)} \theta_{d-2} \int_{0}^{2 \pi} \mathrm{d} \theta_{d-1} \\
= & S_{d-1} \int_{0}^{\infty} \mathrm{d} k k^{d-1} e^{-\beta k^{z}} \int_{0}^{\pi} \mathrm{d} \theta_{1} e^{\mathrm{i} k r \cos \theta_{1}} \sin ^{d-2} \theta_{1} \\
= & (2 \pi)^{d / 2} r^{-(d-2) / 2} \int_{0}^{\infty} \mathrm{d} k k^{d / 2} J_{d / 2-1}(k r) e^{-\beta k^{z}} \\
= & (2 \pi)^{d / 2} r^{-d} \int_{0}^{\infty} \mathrm{d} u u^{d / 2} J_{d / 2-1}(u) e^{-\left(\beta r^{-z}\right) u^{z}}
\end{aligned}
$$

where we used [13, eq. (8.411.7)] in the third line in order to express the last angular integral in terms of a Bessel function $J_{\nu}(w)$ and $S_{d}=2 \pi^{d / 2} / \Gamma(d / 2)$ denotes the surface of the $d$-dimensional sphere. 
In order to display explicitly the space-time scaling of the scaling function $f(\tau, \boldsymbol{r})$, we reexpress $\beta=\alpha \tau$ such that $\alpha=\mathrm{i}^{z-2} /(z \mu)$ and then have

$$
f(\tau, \boldsymbol{r})=\frac{f_{0}(\tau)}{(2 \pi)^{d / 2}} r^{-d} \Lambda\left(\frac{\alpha \tau}{r^{z}}\right)
$$

where the scaling function $\Lambda$ is given by the above integral. Applying scale-invariance, gives an obvious differential equation for the last remaining function, hence $f_{0}(\tau)=f_{(0)} \tau^{d / z}$. Finally, use the expansion of the Bessel function $J_{\nu}(w)=\sum_{n=0}^{\infty} \frac{(-1)^{n}(w / 2)^{\nu+2 n}}{n ! \Gamma(\nu+n+1)}$, in order to convert the scaling function $f(\tau, \boldsymbol{r})=(2 \pi)^{-d / 2} f_{(0)}\left(r^{z} / \tau\right)^{d / z} \Lambda\left(\alpha \tau r^{-z}\right)$ into a series. This gives the final result

$$
f(\tau, \boldsymbol{r})=f_{00} \frac{\Gamma(d / 2)}{\Gamma(d / z)} \sum_{n=0}^{\infty}(-1)^{n} \frac{\Gamma\left(\frac{2 n+d}{z}\right)}{n ! \Gamma\left(n+\frac{d}{2}\right)}\left(\frac{r^{2}}{4(\alpha \tau)^{2 / z}}\right)^{n}
$$

and where $f_{00}$ is a normalisation constant. Clearly, this series has an infinite radius of convergence for $z>1$.

The sought form of the two-point function $F$ is given by eqs. (3.10) 3.29). The special case of even values of $z$ treated above is compatible with this form 5

\section{Spherical model and field-theoretical description}

\subsection{Spherical model with conserved order parameter}

The spherical model was conceived in 1953 by Berlin and Kac as an exactly solvable mathematical model for strongly interacting spins and has proved ever since to be an useful model for trying out more general ideas. It is usually defined in terms of real spin variable $S(t, \boldsymbol{x})$ attached to each site $\boldsymbol{x}$ of the hyper-cubic lattice $\Lambda \subset \mathbb{Z}^{d}$ and depending on time $t$, subject to the mean spherical constraint

$$
\left\langle\sum_{\boldsymbol{x} \in \Lambda} S(t, \boldsymbol{x})^{2}\right\rangle=\mathcal{N}
$$

where $\mathcal{N}$ is the number of sites. The spin Hamiltonian is $\mathcal{H}=-\sum_{(\boldsymbol{x}, \boldsymbol{y})} S_{\boldsymbol{x}} S_{\boldsymbol{y}}$ where the sum is over pairs of nearest neighbours. At equilibrium, this leads to a second-order phase transition with a critical temperature $T_{c}>0$ for all spatial dimensions $d>2$ and where the critical exponents have non-mean-field values for $d<4$, see e.g. [32] and references therein. We consider here a kinetics given by a Langevin equation with a conserved order parameter (model $\mathrm{B}$ in the terminology of [29])

$$
\partial_{t} S(t, \boldsymbol{x})=-\nabla_{\boldsymbol{x}}^{2}\left[\nabla_{\boldsymbol{x}}^{2} S(t, \boldsymbol{x})+\mathfrak{z}(t) S(t, \boldsymbol{x})+h(t, \boldsymbol{x})\right]+\eta(t, \boldsymbol{x}),
$$

where $\mathfrak{z}(t)$ is the Lagrange multiplier fixed by the mean spherical constraint and the coupling to the heat bath with the critical temperature $T_{c}$ is described by a Gaussian noise $\eta$ of vanishing

\footnotetext{
${ }^{5}$ For example, in the case $z=4$, one may separate the sum into even and odd terms. Repeated application of the duplication formula $\Gamma(2 k)=\pi^{-\frac{1}{2}} 2^{2 k-1} \Gamma(k) \Gamma(k+1 / 2)$ shows that the sums arising are exactly of the kind found in (3.18). We have also seen that the form (3.29) has a Fourier transformation, which is not obvious for all solutions in (3.18).
} 
average and variance

$$
\left\langle\eta(t, \boldsymbol{x}) \eta\left(t^{\prime}, \boldsymbol{x}^{\prime}\right)\right\rangle=-2 T_{c} \nabla_{\boldsymbol{x}}^{2} \delta\left(t-t^{\prime}\right) \delta\left(\boldsymbol{x}-\boldsymbol{x}^{\prime}\right)
$$

and $h(t, \boldsymbol{x})$ is a small external magnetic field (required for the computation of the response) [34, 38, 53, 4]. This constitutes a qualitatively reasonable model for the kinetics of phaseseparation, for instance in alloys.

Essentially the same equations can also be used to describe very different physical situations. For example, the growth of interfaces on a substrate with a conservation of particles along the interface is described by the Mullins-Herring equation [44] (or the equivalent Wolf-Villain model [62]) which in our notation is simply given by the Langevin equation (4.2) with $\mathfrak{z}=0$. Several inequivalent variants exist: one may either take the conserved noise (4.3) or else consider the non-conserved centred noise with variance $\left\langle\eta(t, \boldsymbol{x}) \eta\left(t^{\prime}, \boldsymbol{x}^{\prime}\right)\right\rangle=2 T_{c} \delta\left(t-t^{\prime}\right) \delta\left(\boldsymbol{x}-\boldsymbol{x}^{\prime}\right)$, see [50] for details.

Here, we shall concentrate on the Mullins-Herring model with the conserved noise (4.3).

Since correlators and response have already been studied in detail in the literature [34, 38, [12, 53, 4] it is enough to quote the results. For our purposes, we merely need the full spacetime response in the conserved spherical model for $d>4$ or equivalently the Mullins-Herring equation for any $d$, which reads

$$
\begin{aligned}
& R(t, s ; \boldsymbol{r})=\frac{\sqrt{\pi}}{2^{3 d / 2} \pi^{d / 2} \Gamma(d / 4)}(t-s)^{-(d+2) / 4}\left[{ }_{0} F_{2}\left(\frac{1}{2}, \frac{d}{4} ; \frac{r^{4}}{256(t-s)}\right)\right. \\
& \left.-\frac{8}{d} \frac{\Gamma\left(\frac{d}{4}+1\right)}{\Gamma\left(\frac{d}{4}+\frac{1}{2}\right)}\left(\frac{r^{2}}{16 \sqrt{t-s}}\right){ }_{0} F_{2}\left(\frac{3}{2}, \frac{d}{4}+\frac{1}{2} ; \frac{r^{4}}{256(t-s)}\right)\right] .
\end{aligned}
$$

We want to compare this with the $\mathfrak{a g}(d)$-covariant two-point function, as obtained in the previous section from the non-local representation (2.1), with $z=4$. Before, we can do this, however, we still have to show how to relate a dynamical symmetry of a deterministic equation such as (2.16) with the properties of a solution of a stochastic Langevin equation (4.2).

\subsection{Field-theoretical description}

In order to compare co-variant response computed from the non-local representations and derived in section 3, we appeal to standard methods in non-equilibrium field-theory, see e.g. [60, 61] for introductions. Thereby, we shall generalise previous applications of the method [47, 3, 4, 5, 9, 57] to the present case of non-local representations. Consider the Langevin equation

$$
\partial_{t} \phi=-\frac{1}{4 \mu} \nabla_{\boldsymbol{r}}^{2}\left(-\nabla_{\boldsymbol{r}}^{2} \phi+v(t) \phi\right)+\eta
$$

with the conserved centred gaussian noise

$$
\left\langle\eta(t, \boldsymbol{r}) \eta\left(t^{\prime}, \boldsymbol{r}^{\prime}\right)\right\rangle=-\frac{T_{c}}{2 \mu} \nabla_{\boldsymbol{r}}^{2} \delta\left(t-t^{\prime}\right) \delta\left(\boldsymbol{r}-\boldsymbol{r}^{\prime}\right) .
$$


Following [60, 61, 4], the Janssen-de Dominicis action is

$$
\begin{aligned}
\mathcal{J}(\phi, \widetilde{\phi})= & \int \mathrm{d} u \mathrm{~d} \boldsymbol{R}\left[\tilde{\phi}\left(\partial_{u}-\frac{1}{4 \mu} \nabla_{\boldsymbol{R}}^{2}\left(\nabla_{\boldsymbol{R}}^{2}-v(u)\right)\right) \phi\right] \\
& +\frac{T}{4 \mu} \int \mathrm{d} u \mathrm{~d} \boldsymbol{R} \widetilde{\phi}(u, \boldsymbol{R})\left(\nabla^{2} \widetilde{\phi}(u, \boldsymbol{R})\right)+\mathcal{J}_{\text {init }}(\phi, \widetilde{\phi})
\end{aligned}
$$

where

$$
\mathcal{J}_{\text {init }}(\phi, \widetilde{\phi})=\frac{1}{2} \int \mathrm{d} \boldsymbol{R} \mathrm{d} \boldsymbol{R}^{\prime} \widetilde{\phi}(0, \boldsymbol{R})\left\langle\phi(0, \boldsymbol{R}) \phi\left(0, \boldsymbol{R}^{\prime}\right\rangle \widetilde{\phi}\left(0, \boldsymbol{R}^{\prime}\right)\right.
$$

with complete analogy with non-conserved case [40, 47].

Averages of an observable $\mathcal{A}$ are defined as usual by the functional integral

$$
\langle\mathcal{A}\rangle=\int \mathcal{D}[\phi] \mathcal{D}[\tilde{\phi}] \mathcal{A}[\phi] \exp (-\mathcal{J}(\phi, \widetilde{\phi})) .
$$

Next, one decomposes the action into a deterministic part $\mathcal{J}_{0}$ and a noise part $\mathcal{J}_{b}$

$$
\left.\mathcal{J}[\tilde{\phi}, \phi])=\mathcal{J}_{0}[\tilde{\phi}, \phi]\right)+\mathcal{J}_{b}[\tilde{\phi}]
$$

with

$$
\begin{aligned}
& \mathcal{J}_{0}(\phi, \widetilde{\phi})=\int \mathrm{d} u \mathrm{~d} \boldsymbol{R}\left[\tilde{\phi}\left(\partial_{u}-\frac{1}{4 \mu} \nabla_{\boldsymbol{R}}^{2}\left(\nabla_{\boldsymbol{R}}^{2}-v(u)\right)\right) \phi\right] \\
& \mathcal{J}_{b}=\mathcal{J}_{\text {init }}+\mathcal{J}_{\text {th }} ; \quad \mathcal{J}_{\text {th }}=\frac{T}{4 \mu} \int \mathrm{d} u \mathrm{~d} \boldsymbol{R} \widetilde{\phi}(u, \boldsymbol{R})\left(\nabla^{2} \widetilde{\phi}(u, \boldsymbol{R})\right) .
\end{aligned}
$$

The point of this decomposition is that the action $\mathcal{J}_{0}$ is invariant under the non-local representations constructed in section 2. We call the theory with action $\mathcal{J}_{0}$ noise free or deterministic and denote the average with respect to it by $\langle\ldots\rangle_{0}$. Then any average of the full theory can then be computed by formally expending around the noise-free theory

$$
\langle\mathcal{A}\rangle=\left\langle\mathcal{A} \exp \left(-\mathcal{J}_{b}\right)\right\rangle_{0} .
$$

We can focus on the deterministic theory with $v=0$ because of the following observation: If $\phi(t, \boldsymbol{r})$ is a solution of equation (4.5) with $\eta=0$ then the field

$$
\psi(t, \boldsymbol{r}):=\exp \left(\frac{1}{4 \mu} \int_{0}^{t} \mathrm{~d} \tau v(\tau) \nabla_{\boldsymbol{r}}^{2}\right) \phi(t, \boldsymbol{r})
$$

fulfils the same equation (4.5) with $v=0$ (and $\eta=0$ ). Hence, it is enough to consider first the problem with $v=0$ and apply the inverse of the transformation (4.14) for treating the case $v \neq 0$, in order to implement the breaking of time-translation invariance. On the other hand, the case $v=0$ is relevant for $d>4$ in the spherical model and for any $d$ in Mullins-Herring model, which are the cases we are interested in.

The noise-free theory is given by the equation (4.5) with $\eta=0, v=0$ or by the action $\mathcal{J}_{0}$ with $v=0$. This equation coïncides with the equation (2.5) in section 2 with $n=2$ when 
$x+\xi-(d+2) / 2=0$. If the fields $\phi, \widetilde{\phi}$ are indeed quasi-primary, that is they transform under non-local representation (2.1) of the ageing algebra $\mathfrak{a g e}(d)$, it follows that

$$
\phi=\phi_{(\mu, x, \xi)} \quad, \quad \widetilde{\phi}=\widetilde{\phi}_{(-\mu, \tilde{x}, \tilde{\xi})}
$$

are characterised with opposite "mass" parameter and scaling dimensions $(x, \xi),(\tilde{x}, \tilde{\xi})$ correspondingly, with relation

$$
x+\xi=\tilde{x}+\tilde{\xi}=(d+2) / 2 .
$$

The noise-free theory then is invariant under the non-local representation (2.1) of $\mathfrak{a g}(d)$. The Bargman super-selection rule [2] holds in term of the "mass" parameter $\mu$ that is

$$
\left\langle\phi_{1}\left(t_{1}, \boldsymbol{r}_{1}\right) \ldots \phi_{n}\left(t_{n}, \boldsymbol{r}_{n}\right)\right\rangle_{0}=0
$$

unless $\mu_{1}+\ldots \mu_{n}=0$. On the other hand because of the Gaussian structure of the action $\mathcal{J}_{0}$ [4] of the noise-free theory, Wick's theorem applies and gives [64]

$$
\begin{aligned}
& \left\langle\phi_{1}\left(t_{1}, \boldsymbol{r}_{1}\right) \ldots \phi_{2 n}\left(t_{2 n}, \boldsymbol{r}_{2 n}\right)\right\rangle_{0}=\sum_{\text {All possible pairings P of } 1,2, \ldots 2 n} \\
& \left\langle\phi_{P_{1}}\left(t_{P_{1}}, \boldsymbol{r}_{P_{1}}\right) \phi_{P_{2}}\left(t_{P_{2}}, \boldsymbol{r}_{P_{2}}\right)\right\rangle_{0} \ldots\left\langle\phi_{P_{2 n-1}}\left(t_{P_{2 n-1}}, \boldsymbol{r}_{P_{2 n-1}}\right) \phi_{P_{2 n}}\left(t_{P_{2 n}}, \boldsymbol{r}_{P_{2 n}}\right)\right\rangle_{0}
\end{aligned}
$$

As in the non-conserved case [47, 4], it follows that the response function is independent of noise, while in noise-free theory it is given by

$$
R(t, s ; \boldsymbol{x}-\boldsymbol{y}):=\left\langle\phi(t, \boldsymbol{x}) \nabla_{\boldsymbol{y}}^{2} \widetilde{\phi}(s, \boldsymbol{y})\right\rangle_{0}=\nabla_{\boldsymbol{r}}^{2} F^{(2)}(t, s ; \boldsymbol{r})
$$

where $F^{(2)}(t, s ; \boldsymbol{r})$ is the two-point function computed in the previous section in eqs. (3.10) 3.18); after taking time-translation invariance into account, which we achieve under important assumption $x+\tilde{x}=d$, in addition to the relations (4.16). It follows

$$
\begin{aligned}
R(t, s ; \boldsymbol{r})= & (t-s)^{-d / 4} \nabla_{r}^{2} f\left(\frac{\boldsymbol{r}^{4}}{t-s}\right)=(t-s)^{-(d+2) / 4} \Delta_{p} f(p) \\
= & 4(t-s)^{-(d+2) / 4}\left((d+2) p^{\frac{1}{2}} \partial_{p}+4 p^{\frac{3}{2}} \partial_{p}^{2}\right) \times \\
& \times\left[f_{0}{ }_{0} F_{2}\left(\frac{1}{2}, \frac{1}{2}+\frac{d}{4} ;-\frac{\mu p}{64}\right)+f_{1} p^{1 / 2}{ }_{0} F_{2}\left(\frac{3}{2}, \frac{d}{4}+1 ;-\frac{\mu p}{64}\right)\right. \\
& \left.+f_{2} p^{1 / 2-d / 4}{ }_{0} F_{2}\left(1-\frac{d}{4}, \frac{3}{2}-\frac{d}{4} ;-\frac{\mu p}{64}\right)\right] \\
= & (t-s)^{-(d+2) / 4}\left[f_{1}^{\prime}{ }_{0} F_{2}\left(\frac{1}{2}, \frac{d}{4} ;-\frac{\mu p}{64}\right)+f_{0}^{\prime} p^{1 / 2}{ }_{0} F_{2}\left(\frac{3}{2}, \frac{d}{4}+\frac{1}{2} ;-\frac{\mu p}{64}\right)\right. \\
& \left.+f_{2}^{\prime} p^{1-d / 4}{ }_{0} F_{2}\left(\frac{3}{2}-\frac{d}{4}, 2-\frac{d}{4} ;-\frac{\mu p}{64}\right)\right] .
\end{aligned}
$$

While the response function must be regular for $\boldsymbol{r}=0$ and goes to 0 when $\boldsymbol{r} \rightarrow \infty$ the third term is eliminated and the constants $f_{0}^{\prime}$ and $f_{1}^{\prime}$ must be related. Using the known long-term behaviour of the hyper-geometric function [63, 24] we recover exactly the result (4.4).

Therefore, we have seen that the exact result (4.4), rather than being interpreted as in 4 ] a consequence of a local symmetry, the closure of which as a Lie algebra is somewhat artificial and ad hoc, may also be seen as evidence of a non-local representation (2.1) of the well-known Lie algebra $\mathfrak{a g e}(d)$, where the closure is naturally provided by the same equation of motion which is under study! 


\section{Conclusions}

In trying to construct a closed set of Lie algebra generators for generalised scale-transformations with an arbitrary dynamical exponent $z$, we have been led to consider non-local representations of the the ageing algebra $\mathfrak{a g e}(d)$. Clearly, the development of such algebraic techniques can only be a first step to hopefully address in the future the considerably more difficult question of genuinely non-linear equations. In this spirit, the present work is intended as a case study and is meant to provide at least one explicit example. Our construction was guided by the previously known results for $d=1$ space dimensions [24], herein extended to generic $d>1$. It turns out that the result depends on the value of the dynamical exponent $z$.

Conceptually, we have slightly extended the usual definition of the notion of dynamical symmetry, see for example [46]. Conventionally, the infinitesimal generator $X$ of a dynamical symmetry of the equation of motion $\mathcal{S} \psi=0$ must satisfy $[\mathcal{S}, X]=\lambda_{X} \mathcal{S}$ as an operator. Furthermore, it is conventionally admitted that $\lambda_{X}$ should be a scalar or a function. Here, we have allowed for the possibility that it may also become an operator. In our construction of non-local representations of ageing algebra this appears only for the generator of special (conformal) transformations $X_{1}$, for which $\lambda_{X_{1}}$ is a differential operator.

In addition we use a quotient space construction we use to close the algebra. Such a construction appears to be necessary whenever the dynamical exponent $z \neq 1,2$. At least, this appears to be a more elegant and hopefully mathematically more sober way to achieve the closure of the various generators into a Lie algebra, and appears to us preferable to the more traditional approach where several ad hoc super-selection rules had to be imposed in order to cut off the infinite set of non-closing operators, see [18, 4, 22, 23].

Several details depend on the value of $z$

1. For $z$ even, the algebra (2.1) contains the usual local generators of space-translations, spatial rotations, dynamical scaling and global phase shift. In addition, the further $d+1$ generators of generalised Galilei-transformation and special transformations are non-local and are constructed with linear differential operators of order $z-1$. By analogy with the $1 D$ case [24], we suspect that these might be interpreted as generating transformation of distribution functions of the positions, rather than bona fide coordinate transformations. The constructed representation acts naturally as a dynamical symmetry on the solution space of the simple linear Schrödinger equation we considered, and should be viewed essentially as a toy model. The example studied here might be the first step towards an understanding how to use such non-local transformations in applications to the nonequilibrium physics of strongly interacting particles.

We recall that in the context of interface growth with conserved dynamics, exactly the kind of non-local generalised Galilei-transformation we have studied here has already been introduced in analysing the stochastic equation (related to molecular beam epitaxy $(\mathrm{MBE}))$

$$
\partial_{t} \phi=-\nabla^{2}\left[\nu \nabla^{2} \phi+\frac{\lambda}{2}(\nabla \phi)^{2}\right]+\eta
$$

where $\eta$ is a centred and conserved gaussian noise and $\nu, \lambda$ are constants [59]. Indeed, they show that Galilei-invariance leads to a non-trivial hyperscaling relation, expected to be exact and checked in the lowest orders of the loop expansion. In particular, they obtain 
$z=4$ in $d=2$ space dimensions [59]. Similar results exist for slightly different non-linear conserved growth equations [51]. We hope to return to a symmetry analysis of these non-linear equations in the future. In any case, the available evidence that generalised Galilei-invariance could survive the loop expansion is very encouraging.

Since the non-local representations only close over solution of the same equation, we deal with an on shell representation.6 It would be interesting to see whether a systematic relationship with conditional symmetries in partial differential equations can be found, see [7] and references therein.

2. For generic values of $z \geq 2$, the generalisation from the one-dimensional case requires the explicit introduction of some kind of fractional derivative. For our purposes, the Riesz fractional derivative turned out to have the required algebraic properties. In addition, the result derived for the co-variant two-point function is compatible with the directly treatable case when $z$ is even. We are not aware of confirmed physical applications with generic values of $z$. It remains to be seen if similar symmetries of other non-local equations can be found.

On the other hand, the generalisation proposed here in terms of strongly non-local Riesz fractional derivatives into the generators permits at least to close the representation on the solution space of a single partial differential equation. Another advantage of this realisation is that it naturally includes the case $z$ even, when the fractional derivatives become the powers of the Laplacian. It formally can be interpreted as dynamical symmetry algebra and may have unexpected applications which we hope to find in the future.

3. One important difference between $z=2 n$ even and $z$ generic is that in the latter case, the only technique available of solving fractional differential equations, namely by Fourier transformation, gives merely a subset of the solutions which can be found by Frobéniustype series for $z=2 n$.

It remains an open question whether different choices of the fractional derivative are possible, which may also imply that different techniques for finding $n$-point functions will have to be used. Our aim has been to provide at least one worked-out example and we do not claim that the technical choices made here should be unique.

It also remains to be seen how to interpret the 'finite' transformations obtained from the exponentiated non-local generators $\exp (\varepsilon X)$ geometrically.

4. For $1<z<2$, the radius of convergence of the series representations of the two-point function is still infinite and for $z=1$ it is finite. It could be of interest to see whether the specific form (3.29) might already be of relevance in concrete models. Alternatively, it might be conceivable that non-local representations, constructed from the standard local representations of the conformal Galilei algebra (CGA) which have $z=1$ [39, 7] could cover the range $1 \leq z<2$. This remains an open question for future work.

Clearly, the extension of the present method to non-linear equations remains another important open question.

\footnotetext{
${ }^{6}$ Its sub-algebra, however, which includes the dynamical scaling and Galilei algebra is off shell that is, it is closed without any conditions.
} 
Acknowledgements: Most of the work on this paper was done during the visit of S.S. at the Université Henri Poincaré Nancy I (now Université de Lorraine Nancy). The visit was supported in parts: by the Bulgarian NSF grant DO 02-257 and the Université Henri Poincaré Nancy I. M.H. was partly supported by the Collège Doctoral Nancy-Leipzig-Coventry (Systèmes complexes à l'équilibre et hors équilibre) of UFA-DFH.

\section{References}

[1] K. Balasubramanian and J. McGrevy, Phys. Rev. Lett. 101, 061601 (2008).

[2] V. Bargman, Ann. of Math. 56, 1 (1954).

[3] F. Baumann, S. Stoimenov and M. Henkel, J. Phys. A39, 4095 (2006).

[4] F. Baumann and M. Henkel, J. Stat. Mech. P01012 (2007).

[5] F. Baumann, S.B. Dutta, and M. Henkel, J. Phys. A: Math. Gen. 40, 7389 (2007).

[6] A.J. Bray, Adv. Phys. 43, 357 (1994).

[7] R. Cherniha and M. Henkel, J. Math. Anal. Appl. 369, 120 (2010).

[8] L.F. Cugliandolo, in Slow Relaxation and non equilibrium dynamics in condensed matter, Les Houches Session 77 July 2002, J-L Barrat, J Dalibard, J Kurchan, M V Feigel'man eds, Springer(Heidelberg 2003) (cond-mat/0210312).

[9] X. Durang and M. Henkel, J. Phys. A: Math. Theor. 42, 395004 (2009).

[10] C. Duval and P.A. Horváthy, J. Phys. A: Math. Theor. 42, 465206 (2009).

[11] C.A. Fuertes and S. Moroz, Phys. Rev. D79, 106004 (2009).

[12] C. Godrèche, F. Krzakala and F. Ricci-Tersenghi, J. Stat. Mech. P04007 (2004).

[13] I.S. Gradshteyn and I.M. Ryzhik, Table of integrals, series and products, $4^{\text {th }}$ ed., Academic Press (London 1980)

[14] N. Gray, D. Minic and M. Pleimling, arXiv:1301.6368

[15] P. Havas and J. Plebanski, J. Math. Phys. 19, 482 (1978).

[16] M. Henkel, J. Stat. Phys. 75, 1023 (1994).

[17] M. Henkel, Phys. Rev. Lett. 78, 1940 (1997).

[18] M. Henkel, Nucl. Phys. B641, 405 (2002).

[19] M. Henkel and J. Unterberger, Nucl. Phys. B660, 407 (2003).

[20] M. Henkel, T. Enss and M. Pleimling, J. Phys. A Math. Gen. 39, L589 (2006).

[21] M. Henkel, R. Schott, S. Stoimenov and J. Unterberger, Confluentes Mathematici 4, 1250006 (2012). 
[22] M. Henkel and F. Baumann, J. Stat. Mech. P07015 (2007).

[23] M. Henkel and M. Pleimling, Non-equilibrium phase transitions vol. 2: ageing and dynamical scaling far from equilibrium, Springer (Heidelberg 2010).

[24] M. Henkel and S. Stoimenov, Nucl. Phys B847, 612 (2011).

[25] M. Henkel, Causality from dynamical symmetry: an example from local scale-invariance, to appear in the proceedings of AGMP-7 (Mulhouse, France, oct 2011), arXiv:1205.5901

[26] M. Henkel, J.D. Noh and M. Pleimling, Phys. Rev. E85, 030201(R) (2012).

[27] M. Henkel, Nucl. Phys. B869, 212 (2013).

[28] M. Henkel and S. Rouhani, arXiv:1302.7136

[29] P. Hohenberg and B.I. Halperin, Rev. Mod. Phys. 49, 435 (1977).

[30] E.V. Ivashkevich, J. Phys. A: Math. Gen. 30, L525 (1997).

[31] H.-K. Janssen, in G. Györgi et al. (eds), From phase-transitions to chaos, World Scientific (Singapour 1992).

[32] G.S. Joyce, in C. Domb and M. Green (eds), Phase transitions and critical phenomena, vol. 2, Academic Press (London 1972); p. 375

[33] M. Kardar, G. Parisi and Y.-C. Zhang, Phys. Rev. Lett. 56, 889 (1986).

[34] J.G. Kissner, Phys. Rev. B46, 2676 (1992).

[35] J. Krug and P. Meakin, Phys. Rev. Lett. 66, 703 (1991).

[36] J. Lukierski, P.C. Stichel and W.J. Zakrewski, Phys. Lett. A357, 1 (2006); Phys. Lett. B650, 203 (2007).

[37] F. Mainardi and R. Gorenflo, Fractional Calculus and Applied Analysis, 10, 269 (2007).

[38] S.N. Majumdar and D.A. Huse, Phys. Rev. E52, 270 (1995)

[39] D. Martelli and Y. Tachikawa, JHEP 1005:091 (2010). [arXiv:0903.5184]

[40] G.F. Mazenko, Phys. Rev. E69, 016114 (2004).

[41] K.S. Miller and B. Ross, An introduction to the fractional calculus and fractional differential equations, Wiley (New York 1993).

[42] D. Minic and M. Pleimling, Phys. Rev. E78, 061108 (2008).

[43] D. Minic, D. Vaman and C. Wu, Phys. Rev. Lett. 109, 131601 (2012)

[44] W.W. Mullins, in N.A. Gjostein and W.D. Robertson (eds), Metal surfaces: Structure, energetics, kinetics, American Society of Metals (Metals Park (Ohio) 1963).

[45] J. Negro, M.A. del Olmo and A. Rodríguez-Marco, J. Math. Phys. 38, 3786 and 3810 (1997). 
[46] U. Niederer, Helv. Phys. Acta 45, 802 (1972).

[47] A. Picone and M. Henkel, Nucl. Phys. B688 217 (2004).

[48] I. Podlubny, Fractional differential equations, Academic Press (New York 1999).

[49] V. Popkov and G.M. Schütz, J. Stat. Phys. 142, 627 (2011).

[50] A. Röthlein, F. Baumann and M. Pleimling, Phys. Rev. E74, 061604 (2006); Erratum E76, 019901 (2007).

[51] S. Das Sarma and R. Kotlyar, Phys. Rev. E50, R4275 (1994).

[52] S.G. Samko, A.A. Kilbas and O.I. Marichev, Fractional integrals and derivatives: theory and applications, Gordon and Breach (Amsterdam 1993).

[53] C. Sire, Phys. Rev. Lett. 93, 130602 (2004).

[54] D.T. Son, Phys. Rev. D78, 106005 (2008).

[55] H. Spohn, Phys. Rev. E60, 6411 (1999).

[56] S. Stoimenov and M. Henkel, Nucl. Phys. B723, 205 (2005).

[57] S. Stoimenov, Fortschr. Phys. 57, 711 (2005).

[58] L.C.E. Struik, Physical Ageing in Amorphous Polymers and Other Materials, Elsevier (Amsterdam 1978)

[59] T. Sun, H. Guo and M. Grant, Phys. Rev. A40, 6763 (1989).

[60] U.C. Täuber, M. Howard and B.P. Vollmayr-Lee, J. Phys. A38, R79 (2005).

[61] U.C. Täuber, in M. Henkel, M. Pleimling and R. Sanctuary (eds) Ageing and the glass transition, Springer (Heidelberg 2007) (cond-mat/0511743)

[62] D.E. Wolf and J. Villain, Europhys. Lett. 13, 389 (1990).

[63] E.M. Wright, J. London Math. Soc. 10, 287 (1935); and Proc. London Math. Soc. 46, 389 (1940); erratum J. London Math. Soc. 27, 256 (1952).

[64] J. Zinn-Justin, Quantum field-theory and critical phenomena, $4^{\text {th }}$ edition, Oxford University Press (2002). 